\title{
Adiaphora en la Reforma protestante: ¿̨minimalismo doctrinal y neutralidad moral? ${ }^{1}$
}

\author{
Manfred Svensson \\ INSTITUTO DE FILOSOFÍA \\ UNIVERSIDAD DE LOS ANDES
}

\section{INTRODUCCIÓN}

La noción de cosas indiferentes (adiaphora) ha desempeñado un importante papel a lo largo de la historia del pensamiento y constituye un caso significativo de cómo un concepto de la tradición filosófica antigua es adoptado por los cristianos para usos radicalmente distintos de los originales. Originalmente, en efecto, esta noción es utilizada por los estoicos para describir estados de un sujeto (como salud o enfermedad) y objetos externos (pobreza o riqueza), pero en manos de los cristianos pasa muy temprano a ser una herramienta para evaluar las variaciones locales del culto $^{2}$. Más tarde, en los tratados escolásticos sobre la especificación del acto moral, la noción de actos indiferentes desempeñaría también un significativo papel, y acabaría luego extendiéndose de la acción moral y del culto también a las creencias, a la pregunta respecto de si acaso las grandes discusiones doctrinales no son más bien respecto de cuestiones indiferentes. Tal tesis, popularizada por el erasmismo pero de raíz tardomedieval, llegaría a ser un importante componente de las discusiones del siglo XVII sobre la tolerancia. El presente artículo busca establecer el papel desempeñado por esta noción en la Reforma protestante del siglo $\mathrm{XVI}$, uno de los puentes entre el discurso medieval y el nacimiento del pensamiento moderno.

$1 \quad$ El presente trabajo es parte de una investigación financiada por el Fondo Nacional de Desarrollo Científico y Tecnológico, FONDECYT, proyecto Nº 11090189 «La distinción entre cosas indiferentes (adiaphora) y necesarias: un paradigma de la modernidad temprana en la obra de Melanchthon y Locke».

2 Véase, por ejemplo, las cartas 54 y 55 de san Agustín. 
La Reforma protestante del siglo XVI implicó naturalmente cambios significativos no solo para la vida eclesiástica y para la teología, sino de muchos modos también para la relación que los forjadores y herederos de dichas reformas habrían de tener con la tradición intelectual precedente. Naturalmente, las interpretaciones contemporáneas de dicho fenómeno fluctúan a lo largo de un amplio espectro: desde quienes ven en la Reforma una ruptura con la tradición intelectual precedente y el primer germen del pensamiento moderno (sea este valorado positiva o negativamente) ${ }^{3}$, hasta quienes acentúan más bien los elementos de continuidad, continuidad por una parte entre la tradición intelectual medieval y el movimiento de Reforma, por otra parte entre la propia Reforma y la posterior escolástica protestante ${ }^{4}$. La noción de cosas indiferentes es un apto punto para abordar este arduo problema. Pues no solo se encuentra presente en un número muy considerable de las controversias de dicho siglo, sino que se encuentra tan presente en las controversias ad extra como en las controversias intraprotestantes. Considérese los siguientes campos de conflicto en que la idea de cosas indiferentes parece encontrar cabida. En primer lugar, se encuentra el sencillo hecho de que las distintas corrientes de la Reforma tienen una voz común en su apelación a las palabras de Cristo en Mateo 15, 9; que contrastan la doctrina de Dios con los «mandamientos de hombres». La crítica a las "ceremonias» que surge de ahí presenta sin duda una amplia variedad, desde la violenta erradicación de todo lo que pareciera mandato de hombres en manos de algunos movimientos radicales, hasta el extremo opuesto de su conservación, pero en dicho caso en calidad de cosas indiferentes. En segundo lugar, el protestantismo bien puede ser caracterizado por cierta concentración doctrinal: hay un singular esfuerzo por ser enfáticos respecto de cuál es el centro de la fe cristiana, cuál es el articulus stantis et cadentis ecclesiae. La centralidad dada al artículo de la justificación, por el que todo cae o permanece en pie, podría, si

3 Se trata de una interpretación frecuente entre pensadores católicos. Véase, por ejemplo, J. Maritain, Tres reformadores: Lutero, Descartes, Rousseau (Encuentro, Madrid, 2006).

4 Para tal lectura véase R. Muller, Post-Reformation Reformed Dogmatics. IV (Baker Academic, Grand Rapids, 1987-2003); W. J. van Asselt, Introduction to Reformed Scholasticism (Reformation Heritage Books, Grand Rapids, 2011); C. Trueman y R. S. Clark (eds.) Protestant Scholasticism. Essays in Reassesment (Paternoster, Carlisle 1999). 
no existen los resguardos necesarios, conducir a un pronunciado minimalismo doctrinal, y cabe por tanto preguntarse si en los reformadores mismos existen tales resguardos, si acaso la concentración doctrinal está desarrollada de un modo que no vuelva indiferentes las creencias que no constituyen el centro del misterio cristiano. En tercer lugar, está el hecho de que precisamente por encontrarse el centro en el artículo de la justificación por la fe, y no en otro, se abre la puerta a que el protestantismo sea acusado de indiferentismo moral. Tal acusación no solo ha hecho prodigiosa carrera en los siglos posteriores, sino que se encuentra presente desde el primer momento y, como veremos, precisamente acudiendo al lenguaje técnico de la indiferencia. Sea que se hable sobre el culto, la doctrina o la moralidad, la discusión sobre la eventual indiferencia no es nada indiferente.

En contraste con la literatura secundaria que ha precedido a nuestra investigación, literatura que ha abordado la discusión sobre la noción de adiaphora en el siglo XVI centrándose de modo casi exclusivo en su uso en el culto ${ }^{5}$, el presente artículo busca abordar el segundo y el tercero de los problemas que acabamos de mencionar: la cuestión de si acaso en la Reforma protestante puede verse un germen de indiferentismo moral, y la cuestión del papel de la misma en la historia del minimalismo doctrinal. En gran medida dejamos pues de lado la discusión sobre el culto. Pero tal limitación de nuestra investigación no nace de una minimización del culto. Por el contrario, la mayor parte de la controversia del siglo XVI en torno a la noción de adiaphora se concentra en dicho campo. En efecto, tras la derrota militar de la liga de Esmalcalda, el Interim de Ausburgo promovido por Carlos V implicaba la imposición imperial de aspectos ceremoniales, provocando una abundante literatura sobre la noción de adiaphora ${ }^{6}$. El adecuado tratamiento de dicha controversia

5 Una representativa muestra de dicha aproximación se encuentra en B. VERKAMP, The Indifferent Mean. Adiaphorism in the English Reformation to 1554 (Ohio University Press, Athens 1977). Curiosamente, la excepción a esta norma se encuentra en una obra de estética, pero que discute abundante material adiaphorista del siglo XVI en el contexto moral y doctrinal: R. SDzuj, Adiaphorie und Kunst. Studien zur Genealogie ästhetischen Denkens (Max Niemeyer Verlag, Tübingen 2005).

6 Una representativa cantidad de la literatura de controversia del período se encuentra reunida en I. Dingel (ed.), Reaktionen auf das Augsburger Interim: der interimistische Streit (1548-1549) (Vandenhoeck \& Ruprecht, Göttingen 2010). Para el análisis del contexto histórico y de la literatura polémica de los distintos 
implicaría también abordar algunos de los primeros desarrollos de una teoría de la resistencia entre los protestantes. La presente investigación no solo omite eso por las proporciones que adquiriría la discusión. El motivo principal es más bien que al abordar en el presente artículo el problema del minimalismo doctrinal y luego el del indiferentismo moral, cabe sacar a la luz el modo en que las discusiones sobre adiaphora en el culto están enraizadas no simplemente en distintas visiones de la liturgia, sino en modos más generales de pensar sobre aquello que es indiferente, o en aquello que es de importancia menor sin deber ser calificado de indiferente.

\section{Minimalismo Doctrinal}

a) Lutero y la assertio contra el minimalismo doctrinal erasmista

Bajo el rótulo de «minimalismo doctrinal» tratamos aquí una tendencia que en gran medida podemos remontar al período tardomedieval, tanto a la devotio moderna como a algunos representantes de la filosofía política del período, que se unen en enfatizar el carácter práctico de la religión, y esto de un modo que no solo tiende a poner la praxis por sobre la doctrina, sino también produciendo modificaciones internas del campo doctrinal, particularmente en términos de una reducción de las doctrinas a las menos conflictivas ${ }^{7}$. Tal tendencia, asociada a planes de pacificación o concordia como los de Nicolás de Cusa, se volvería por supuesto muy significativa en el discurso de la modernidad temprana respecto de la concordia posterior a las guerras de religión. Se trata, en efecto, de un elemento característico del discurso teológico de autores como Hobbes, Spinoza y Locke ${ }^{8}$. Pero no solo se volvería un elemento significativo dentro de la filosofía política moderna, sino que también ha pasado a ser uno de los elementos característicos de una parte signi-

grupos I. Dingel y G. Wartenberg, Politik und Bekenntnis. Die Reaktionen auf das Interim von 1548 (Evangelische Verlagsanstalt, Leipzig 2006).

7 Para la presencia de nuestro tópico en la espiritualidad del período cf. G. Evans, "Sancta Indifferentia and Adiaphora. "Holy Indifference" and "Things Indifferent"”, en Common Knowledge 15/1 (2009), 23-38.

8 Para Hobbes véase Leviatán III, 34, 13; 36, 20; 42, 13 y 29; y, finalmente, 43, 19. Para Spinoza principalmente el capítulo del Tractatus Theologico-Politicus y para Locke el apéndice sobre la herejía en la Epistola de Tolerantia así como The Reasonableness of Christianity. 
ficativa del mundo evangélico contemporáneo, fuertemente centrado en la evangelización, pero reduciéndola muchas veces a una confesión de Jesús como Mesías asombrosamente similar en su minimalismo al tipo de fórmula doctrinal que podía promover Hobbes? ${ }^{9}$ Dadas estas asociaciones tanto con la filosofía política moderna como con el discurso evangélico contemporáneo, resulta de primera importancia establecer la relación de la Reforma protestante con este tipo de discurso.

Hay, en efecto, una popular interpretación del protestantismo que lo ve como una vuelta a una fe sencilla y práctica de los orígenes. Pero cabe notar que si un hombre del siglo XVI hubiese escuchado un llamado de esa naturaleza, lo más probable es que habría vinculado solo uno de esos elementos al protestantismo, el esfuerzo por una vuelta a las fuentes. Pues el otro llamado, el llamado a entender el cristianismo como algo práctico y sencillo, es algo que todo hombre culto del período habría sabido relacionar no con las diversas reformas protestantes, sino con otra de las grandes corrientes del siglo: el humanismo de inspiración erasmista. Pues es en la obra de Erasmo donde se encontrará el llamado a buscar una philosophia Christi que es sencilla, que consiste principalmente en un buen vivir, que está alejada de las disputas escolásticas y del «maximalismo» doctrinal nacido y retroalimentado por tales disputas. El contraste con la posición de los reformadores protestantes salta a la vista cuando Erasmo concede que la fuente de tal filosofía debe encontrarse en las Escrituras apostólicas y evangélicas, pero que también estas son en realidad demasiado difíciles. Lo que se requiere es que hombres eruditos las lean y presenten una síntesis en que «las cosas pertenecientes a la fe deberían ser resueltas en poquísimos artículos» ${ }^{10}$. Si hay en el siglo XVI alguien cuya obra constituye más allá de toda duda un eslabón en la cadena del minimalismo doctrinal, parece pues ser Erasmo. Que su obra influye de modo significativo en la teoría política del siglo XVII también parece ser indubitable ${ }^{11}$. Nuestra pregunta puede pues ser abordada preguntando por la relación de la Reforma con Erasmo.

9 Respecto del minimalismo doctrinal en el mundo evangélico contemporáneo cf. D. G. HART, The Lost Soul of American Protestantism (Rowman \& Littlefield, Lanham 2004).

10 Erasmo de Rotterdam, Epistola ad Paulum Volzium en Ausgewählte Schriften. I (Wissenschaftliche Buchgesellschaft, Darmstadt 1968), 12-14.

11 Véase J. Marshall, John Locke. Resistance, Religion and Responsibility (Cambridge University Press, Cambridge 1994), 5. 
Ahora bien, que hay una general influencia del movimiento humanista sobre todo el siglo XVI, y en particular sobre algunos de los reformadores, es algo que se encuentra fuera de discusión. Aquí conviene pues plantear la cuestión del modo más específico posible, y para esto conviene en primer lugar atender a la controversia de Lutero con Erasmo. A nadie se escapa la relevancia de esta para el rumbo definitivo de la Reforma y el distanciamiento de la misma respecto del resto del movimiento humanista. Con todo, su importancia suele ser reducida a la discusión sobre la libertad de la voluntad humana. Pero si bien ese es efectivamente el objeto principal del De Servo Arbitrio, el prólogo de Lutero a dicha obra le da a esta discusión un significado mucho más amplio, el cual parece tornar la obra importante para lo que aquí discutimos. Pues Lutero se vuelve ahí contra la posición neutral o escéptica con la que caracteriza a Erasmo (venis medius) ${ }^{12}$. En respuesta a la posición de Erasmo según la cual este preferiría suspender el juicio en materias periféricas, Lutero afirma que tampoco respecto de cuestiones menores le cabe al cristiano «dejar de gozarse en las assertiones. Por el contrario, precisamente en las assertiones hay que encontrar gozo, o no se será cristiano» ${ }^{13}$. La assertio podría traducirse de distintos modos: afirmaciones específicas, creencias robustas o juicios categóricos. Pero afortunadamente, en este caso Lutero da una explicación que para nuestro presente propósito es suficientemente clarificadora. En efecto, "para que nadie juegue con palabras", se apura aquí en afirmar que bajo assertio entiende «adherir de modo constante a una doctrina, reforzarla, confesarla, defenderla y aferrarse de modo inconmovible a ella» ${ }^{14}$. Esto no deja mucho lugar a dudas respecto del modo o la tenacidad de la adhesión. Pero por supuesto podría tratar-

12 WA 18, 605. Para las obras de los reformadores protestantes se citará a continuación según las siguientes ediciones. Lutero, Werke. Weimarer Ausgabe [abreviado WA] (Böhlau, Weimar, 1883-). Calvino, Ioannis Calvini opera quae supersunt omnia [CO]. Wilhelm Baum, Eduard Cunitz y Eduard Reuss (eds.) (Braunschweig, 1863-1900). Para su Institución [Inst.] seguimos la edición de Peter Barth et al. en Johannis Calvini Opera Selecta (Chr. Kaiser, Múnich 1926-1952). Melanchthon, Melanchthons Werke in Auswahl [MWA], ed. R. STupperich (Gütersloher Verlagshaus Gerd Mohn, Stuttgart-Bad Cannstatt, 1951-1975) y Opera quae supersunt Omnia en Corpus Reformatorum [CR], ed. C. B. BrettschneIder y H. E. Bindseil (C. A. Schwetschke, Halle-Braunschweig 1834-1860).

13 WA 18, 603.

14 WA 18, 603. Hay una razón más específica por la que este es el término escogido aquí. Cuando Erasmo publica en 1524 su Diatriba sobre el libre albedrío, el texto 
se todavía de un mínimo doctrinal al que se adhiera con tal tenacidad. Sin embargo, en el mismo lugar Lutero precisa dicho contenido no por referencia a alguna doctrina en particular, sino a «lo que Dios nos ha transmitido en las Sagradas Escrituras» ${ }^{15}$. La primera impresión que deja este prólogo es, en efecto, de una considerable robustez, no solo en el tono de Lutero, sino en la amplitud de lo confesado.

Lutero concede luego que puede haber ideas sin importancia o indiferentes (inutilia et neutra dogmata) ${ }^{16}$, y que crear conflicto respecto de ellas puede ser impío. Pero lo decisivo es que el campo de lo que debe ser objeto de consideración, adhesión y contienda no es la doctrina de la justificación, sino el conjunto del testimonio bíblico o, como lo precisarían otros protestantes mediante una expresión paulina, «el consejo total de Dios» (Hch 20, 27). Algo más adelante, en efecto, Lutero amplía esta afirmación, sosteniendo que un cristiano «no solo querrá volverse firme mediante la Escritura y adherir a ella en todo y en cada punto (ubique in omnibusque partibus), sino que también querrá alcanzar la mayor firmeza posible en aquello que no es necesario y que se encuentra fuera de los márgenes de la Biblia (extra scripturam)» ${ }^{17}$. Estamos pues ante una preocupación tanto por la amplitud de lo que debe ser confesado -incluyendo en ello conocimiento extrabíblico-, como por la precisión con que esto debe ocurrir. Y este tipo de cuidado por la precisión doctrinal es aún más notorio en el resto de los reformadores. En su crítica a aquellos teólogos alemanes que aceptaron el ya mencionado Interim impuesto por Carlos V, Calvino escribe -en un tono muy similar al reproche de Lutero a Erasmo- contra los «falsos mediadores que imaginan una vía media» ${ }^{18}$. Pero lo que Calvino opone a dicha vía media no es el camino de un simple extremismo, sino que es un llamado a la precisión doctrinal. Su advertencia abunda, en efecto, en exhortaciones a definiciones precisas en lugar de una paz buscada con generalidades que oculten los puntos en controversia: hay una gran diferencia, escribe, entre afirmar que se cree en la justificación por la fe, y dar una explicación exacta de

de Lutero que toma como punto de referencia para iniciar la discusión es la Assertio omnium articulorum, con la que Lutero responde en 1520 a León X.

15 WA 18, 603.

16 WA 18,604 .

17 WA 18, 604.

$18 \mathrm{CO} 7,603-4$. 
la cuestión ${ }^{19}$. De modo análogo, casi un siglo y medio más tarde, John Owen escribiría advirtiendo contra predicadores que se conforman con "principios generales» en lugar de "explicar, confirmar y vindicar cada verdad que hemos recibido» ${ }^{20}$. A la luz de lo que aquí hemos visto, tal búsqueda de precisión doctrinal, la preocupación por lo que a algunos pueden parecer sutilezas, no es un abandono de la Reforma impulsado por la escolástica predominante en la generación de Owen, sino que se encuentra en plena continuidad con el impulso original. Las palabras de Owen son, de hecho, casi idénticas con la explicación de Lutero respecto de lo que entiende bajo una assertio.

Esto debería volvernos cautos respecto de entender la doctrina de la justificación como el artículo necesario, y otros artículos en cambio como indiferentes. Pues si bien, como hemos visto, Lutero afirma en un comentario marginal la existencia de ciertos inutilia et neutra dogmata, tal expresión parece un modo impropio de hablar, incluso si es medida por la vara del propio Lutero. Porque al abordar de modo detenido la idea de una distinción entre doctrinas necesarias y no necesarias, esto es algo que Lutero denuncia como una posición erasmista ${ }^{21}$. El motivo por el que Lutero rechaza tal distinción guarda relación con el hecho de que aquí se enfrentan visiones rivales de las Escrituras, visiones que aquí tiene sentido, aunque sea brevemente, explicitar. La distinción erasmistas entre lo necesario y lo no necesario puede considerarse como caracterizada por dos distinciones paralelas: la de lo teórico y lo práctico, y la de lo oscuro y lo claro. Las cosas necesarias son para Erasmo cosas prácticas respecto de las cuales la Biblia es clara; las cosas indiferentes son cosas teóricas respecto de las cuales la Biblia es oscura. Una de estas parejas conceptuales paralelas -la distinción entre lo teórico y lo prácti-

19 CO 7, 599. Iam satis patere arbitror, quantopere referat, ut salva maneat de iustificatione doctrina, habere certam fidei definitionem. CO 7, 594. Deinde multum interest, verbone uno duntaxat proferas, fide nos iustificari, an rem totam diserte explicatam proponas.

20 J. Owen, A Discourse Concerning Evangelical Love, Church Peace, and Unity in The Works of John Owen, XV (Banner of Truth Trust, Londres 1965), 109.

21 WA 18, 606. dogmata Christiana distinguis, quaedam scitu necessaria, quaedam non necessaria fingis. Sobre el carácter erasmista de este lenguaje, Lutero tiene toda la razón. Incluso la idea de un articulus stantis et cadentis eclessiae, típicamente vinculada a Lutero, es formulada primero por Erasmo, como lo ha hecho notar H. Reventlow, The Authority of the Bible and the Rise of the Modern World (SCM Press, Londres, 1984), 48. 
co- no es abordada por Lutero en el contexto de esta polémica. La otra distinción, en cambio, sí es uno de los tópicos principales de discusión del De servo arbitrio, donde Lutero busca defender que también para materias específicas como la relación entre libre albedrío y gracia, la $\mathrm{Bi}$ blia es suficientemente clara. El modo en que estos distintos tópicos se entrelazan en la disputa nos permite hacer una significativa aclaración. En la tradición posterior con frecuencia se ha pensado como si el énfasis protestante en la perspicuitas de las Escrituras fuera un indicio de que la Reforma se encontrara alineada con un énfasis erasmista en la sencillez del mensaje cristiano. Pero tal vez la situación sea la inversa: Erasmo cree en un mensaje sencillo y práctico, pero no considera la claridad como uno de los atributos principales de las Escrituras; los reformadores protestantes creen en tal claridad, pero no parecen ser expositores de un mensaje práctico y sencillo. Identificar perspicuitas con sencillez del mensaje parece entonces ser fatal para la comprensión de la controversia teológica del siglo XVI.

Ahora bien, nada de lo precedente implica por parte de Lutero un rechazo a una concepción gradual o jerarquizada de las doctrinas. De hecho, los artículos de Esmalcalda, cuyo redactor principal es Lutero, están concebidos en términos de una cierta hierarchia veritatum, por usar la expresión del Vaticano II ${ }^{22}$. Pero sí está implicado rechazar la idea de que haya un conjunto de doctrinas indiferentes. De la posición de Erasmo Lutero se considera separado por un abismo más grande que el que lo separa de la escolástica medieval ${ }^{23}$. En efecto, interpreta esta distinción entre dos tipos radicalmente distintos de doctrinas, una de las cuales sería tan indiferente que podemos ser escépticos respecto de ella, como un camino de Erasmo para acabar reclamando libertad también respecto de las doctrinas que originalmente considera necesarias ${ }^{24}$. Anticipándose a la crítica que un siglo más tarde harían los escolásticos reformados al minimalismo doctrinal de sus contemporáneos ${ }^{25}$, Lutero escribe que

22 Unitatis Redintegratio, n. 11. La adopción de esta fórmula ha tenido de hecho influencia retrospectiva en el estudio de importantes pensadores protestantes. Véase, por ejemplo, F. Duchesneau, «Leibniz: La bible et l'ordre des vérités», en Revue de théologie et de philosophie 133, 3 (2001) 267-286.

23 WA 18, 613. mitius tamen peceant quam tu.

24 WA 18, 604.

25 Por ejemplo, H. Witsius, Excercitationes sacrae in symbolum quod apostolorum dicitur (Johannem Wolters, Amsterdam, 1697) II, 6. 
el cristianismo profesado por Erasmo es algo que «cualquier judío o pagano ${ }^{26}$ podría profesar, de hecho algo que ni siquiera del judaísmo deja algo en pie $^{27}$. Su inequívoca conclusión -expresada dos veces en este prólogo- es que esto ya es ateísmo ${ }^{28}$. Apenas se podría encontrar un testimonio más elocuente de la distancia entre la Reforma protestante y la reforma humanista respecto de la materia que aquí consideramos.

b) Melanchthon y la recepción del método de los loci

En un segundo paso nos detendremos en Melanchthon, concentrándonos en la cercanía o distancia respecto de Erasmo en términos metodológicos. Pues el sistema de los loci, presentado por Erasmo en su De copia en 1512, parece ser precisamente una materialización de la mentalidad que ya hemos descrito: se lee el texto bíblico buscando sacar de él un conjunto de "tópicos fundamentales», y teniendo esos tópicos se va añadiendo a estos el fruto de la continuada lectura. Este el sistema adoptado por Melanchthon en la primera dogmática luterana, los Loci Communes de 1521, una obra cuya relevancia metodológica no solo sobre el luteranismo, sino sobre el calvinismo y sobre el mismo Calvino es crecientemente reconocida ${ }^{29}$. Con todo, antes de abordar el modo en que este método se vincula o no a un proyecto doctrinalmente minimalista, cabe decir algo preliminar sobre el eventual carácter intermedio de Melanchthon como figura entre Erasmo y Lutero. Tal fue por mucho tiempo el modo convencional de leerlo, una lectura que en consecuencia veía el actuar de Melanchthon durante el Interim, y su posición respecto de las cosas indiferentes, como un ejemplo de moderación erasmista. Franz Hildebrandt, el destacado colaborador de Bonhoeffer, es quien tal vez más lejos ha ido a este respecto, escribiendo que Melanchthon se sitúa "en la gran tradición humanista» de gente como Calixto y Grocio, que busca "la esencia del cristianismo» más allá de las diferencias

\footnotetext{
WA $18,609$.

WA 18,610 .

WA 18,605 y 611.

R. Muller, "To Elaborate on the Topics: The Context and Method of Calvin's Institutes", en Muller The Unaccomodated Calvin. Studies in the Foundation of a Theological Tradition (Oxford University Press, Oxford, 2000), 101-117. Véase asimismo las diversas contribuciones en G. Frank - H. Selderhuis, Melanchthon und der Calvinismus (frommann-holzboog, Stuttgart- Bad Cannstatt 2005).
} 
confesionales y que declara libertad respecto de todo lo no esencial ${ }^{30}$. Tal actitud, la disposición a ceder respecto de cuestiones que pudiesen parecer secundarias para la esencia del cristianismo, había sido reconocida como particularmente fatal por parte de quienes en la generación de Hildebrandt y Bonhoeffer tenían que justificar teológicamente la resistencia ante el nacionalsocialismo. Hoy, aunque expresada en términos más cautos, esta sigue siendo una común concepción de Melanchthon ${ }^{31}$. Con todo, el estudio sobre su obra en las décadas recientes ha llevado a que toda la idea de Melanchthon como figura intermedia se encuentre profundamente puesta en duda, principalmente por las investigaciones de Timothy Wengert, que muestran a alguien de principio a fin mucho más decididamente distanciado de Erasmo ${ }^{32}$. A la luz de eso, cabe una revisión de su posición respecto de las cosas indiferentes ${ }^{33}$.

Si partimos por una comparación con sistemas teológicos del tipo de las Summae medievales, podemos decir que estas aspiraban no solo a una exposición sistemática de todo el cuerpo de la doctrina cristiana, sino también a establecer la relación entre sus distintos elementos. Ambos pasos contribuyen a que no haya doctrina alguna que pueda ser vista como indiferente. El sistema de los loci parece empujar en una dirección distinta, al buscar la concentración en ciertos «tópicos fundamentales». En efecto, se trata de un lenguaje que parece de suyo invitar a la concentración en lo esencial o en lo prácticamente decisivo. Pues los loci de 1521 pueden ser clasificados como erasmistas no solo por el énfasis en ciertos pocos loci, sino por el modo en que precisamente los aspectos más especulativos de la teología tienden a quedar en el bloque de lo

30 F. Hildebrandt, Melanchthon, Alien or Ally? (Cambridge University Press, Cambridge 1946), 82.

31 Para un caso reciente puede verse la imagen de Melanchthon transmitida en O. Olson, Matthias Flacius and the Survival of Luther's Reform (Lutheran Press, Minneapolis 2011). La obra pasa por alto las investigaciones de Wengert que mencionamos a continuación.

32 Véase principalmente T. Wengert, Human Freedom, Christian Righteousness. Philip Melanchthon's Exegetical Dispute with Erasmus of Rotterdam (Oxford University Press, Oxford 1998).

33 Esto es un trabajo que respecto del grueso de la obra de Melanchthon puede aún considerarse pendiente, aunque el mismo Wengert ha dado un primer paso con su evaluación de la primera reacción de Melanchthon al Interim en «Not by Nature Philoneikos. Philip Melanchthon's Initial Reactions to the Augsburg Interim, en I. Dingel - G. Wartenberg, Politik und Bekenntnis, 33-50. 
indiferente. El considerar lo teórico como indiferente, es, como ha sido mencionado, uno de los rasgos más típicos de la tradición erasmista ${ }^{34}$, y los loci de Melanchthon son célebres por su afirmación de que «haremos mejor en adorar que en investigar los misterios de la divinidad ${ }^{35}$. Parece indiscutible que en un primer momento hay una tendencia a tratar la teología especulativa como algo indiferente. En ese sentido hay una similitud estructural con la posición de Erasmo. Pero solo estructural, pues los contenidos que llenan esta estructura no admitirían concordia entre Erasmo y Melanchthon: las cosas que Melanchthon deja en el lado práctico, como necesarias para la salvación y objeto por tanto de la exposición teológica, siguen siendo cosas que Erasmo pasaría al campo de lo indiferente; de ahí el irrestricto apoyo de Lutero a esta obra de Melanchthon $^{36}$. La idea de una inclinación del método al minimalismo doctrinal también es algo respecto de lo cual se puede plantear algunas dudas: si bien parece existir tal tendencia, ya en los loci de $1521 \mathrm{Mel}-$ anchthon expresa su posición diciendo que se centrará en los loci principales, «de los que pende el resto del conjunto (rerum summa) » ${ }^{37}$; se trata de una frase reveladora en cuanto establece una cierta jerarquía doctrinal, que pone a ciertos artículos como más decisivos, pero al mismo tiempo reconoce que hay algo más, algo que pende de ellos.

Con todo, lo más decisivo es que la posición que acabamos de reseñar es una que solo caracteriza al Melanchthon de la primera edición de los loci. Esta obra pasaría no solo por cuantiosas revisiones en cada reimpresión, sino por dos redacciones completamente distintas, los loci secundae aetatis (base de las diversas ediciones de 1535-1543) y tertiae aetatis (1543-1559). Al preparar la edición para el Corpus Reformatorum, Bindseil llega a contar 75 distintas ediciones de la pluma del mismo Melanchthon ${ }^{38}$. Y lo que aquí nos interesa al respecto, es el hecho de que dichos cambios implicaran una creciente robustez doctrinal. La causa de tal evolución se ha puesto frecuentemente en el enfrentamiento con los grupos radicales que arribaron a Wittenberg en 1521 y la posterior

\footnotetext{
34 Véase G. Remer, Humanism and the Rhetoric of Toleration (Pennsylvania State University Press, University Park 1996), 74-75.

35 MWA II/1, 19.

36 WA $18,601$.

37 MWA II/1, 19.

38 CR 21, 59-72, 231-42, 563-94.
} 
guerra de los campesinos en 1525. En efecto, la importancia de tales fenómenos para la evolución de Melanchthon no puede ser subestimada. Pero debe al mismo tiempo tenerse presentes las controversias algo más silenciosas que a lo largo de los años veinte mantiene con humanistas como Erasmo o con antinomistas como Johann Agricola. La diferencia que el conjunto de estas controversias genera respecto de su posición más temprana se puede constatar dirigiendo la mirada a la "epístola al lector» con que se abre la última edición, de 1559. Mientras la dedicatoria de 1521 introduce una obra sobre los tópicos principales (praecipui loci) ${ }^{39}$, la de 1559 parte hablando sobre lo importante que es familiarizarse no con los principales, sino con cada tópico de la doctrina cristiana (singulis articulis doctrinae Christianae) ${ }^{40}$. Como ha sido notado en más de una ocasión, esto efectivamente se traduce en una mucho mayor preocupación por los artículos «teóricos», como puede verse de modo primordial en la ampliada discusión sobre la doctrina trinitaria en su obra madura ${ }^{41}$. Esto, por lo demás, no es algo que se encuentre solo en medio de sus obras dogmáticas, sino algo que tendrá presente como defensa en medio de la controversia adiaphorista tras la muerte de Lutero. Acusado por las concesiones del Interim, apela precisamente apuntando al tipo de sistema comprehensivo (totum doctrinae corpus) que ha intentado exponer y defender una y otra vez ${ }^{42}$. La obra de Melanchthon, quien inicialmente parecería un mejor candidato que Lutero para un vago erasmismo que separe doctrinas «fundamentales» de un resto «indiferente», más bien confirma la oposición entre los dos tipos de reforma.

c) Construyendo sobre el fundamento: Calvino sobre 1 Corintios 3, 15

Completaremos este lado de la discusión dirigiendo la mirada al modo en que es comentado un pasaje bíblico que se volvería predilecto de los minimalistas doctrinales del siglo XVII, esto es, 1 de Corintios 3, 1015, con su llamado a cuidar la materia con que se construye sobre el fundamento que es Cristo, pues lo sobreedificado tendrá que pasar por fuego. Avanzado el siglo XVII, este pasaje sería típicamente usado como

39 MWA II/1, 17.

40 MWA II/1, 186.

41 Véase S. Grosse, «Melanchthons Wende zut Trinitätslehre», en Kerygma und Dogma 54, 4 (2008), 264-289.

42 CR 9, 91. 
prueba textual de la idea de que hay ciertas doctrinas fundamentales, las únicas necesarias para la salvación, y que de otras doctrinas se podría prescindir. El pasaje, utilizado de este modo, no apoya la idea de una jerarquía de doctrinas, sino más bien la separación entre las necesarias y las indiferentes. Con todo, no solo esto parece problemático, sino que la idea misma de aplicar el pasaje de modo tan inequívoco a las doctrinas parece, a luz de la historia de la exégesis del pasaje, algo digno de cierta reflexión. En efecto, si dirigimos la mirada a la historia de interpretación del pasaje, se puede constatar que en un comienzo su interpretación es variada. Clemente de Alejandría lo lee como una clara referencia a la legitimidad de una profundización doctrinal: el fundamento es la fe sencilla, y sobre esta los herejes construyen paja mientras el sabio cristiano -el «verdadero gnóstico»- construye con oro y piedras preciosas ${ }^{43}$. San Agustín, en cambio, en su de fide et operibus ${ }^{44}$, interpreta el pasaje con una clara aplicación a la vida práctica, no a la doctrina: el oro y la paja son posibles tipos de acciones, de modo que el pasaje es interpretado como un llamado a construir con acciones que sean materiales sólidos, compatibles con la solidez del fundamento que es Cristo. Así, no es de extrañar que Tomás de Aquino expusiera el pasaje como susceptible de ambas interpretaciones, como si se sobreedificara con doctrinas o con obras; se abstiene, en efecto, de indicar cuál de estas considera correc$\mathrm{ta}^{45}$. Ahora bien, en el siglo XVI se está ante una coyuntura que parece invitar a interpretarlo con un énfasis en la doctrina, pues esto permitiría a los reformadores protestantes interpretar la posición católico-romana como una que habría «sobreedificado» con material incompatible con el fundamento ${ }^{46}$. Después de todo, una crítica frecuente en el período es la que se fija no solo en el modo en que ciertas doctrinas son comprendidas, sino aquella según la cual el catolicismo romano ha añadido «tradiciones de hombres» a lo prescrito por Dios, la idea de que ha habido una proliferación de credenda. Con todo, cabe notar que este tipo de exposición del pasaje es menos frecuente entre los reformadores que lo que cabría esperar. Dirigirnos a la exposición que Calvino hace del

43 Clemente, Stromata V, 26, 1-5.

44 Agustín, De fide et operibus I, 1.

45 Tomás de Aquino, Super I Cor., cap. 3 1. 2.

46 Véase I Backus, Historical Method and Confessional Identity in the Era of the Reformation (1378-1615) (Brill, Leiden 2003), 256. 
mismo confirma, por el contrario, la distancia entre la Reforma y el minimalismo doctrinal.

Para constatar esto hay que dirigir la mirada a su comentario a 1 de Corintios. Ahí Calvino lee el pasaje viendo la "paja» y el «oro» como doctrinas sobreedificadas, pero aplica este modo de leerlo no a las doctrinas contenidas en la Biblia, sino a las doctrinas contenidas entre los comentadores de la misma: es en ellos que puede haber «sobreedificaciones» tanto útiles como inútiles ${ }^{47}$. Pero el fundamento sobre el cual se realiza dicha sobreedificación no es un mínimo. Es Cristo, pero Calvino se apura en conectar 1 de Corintios con la afirmación paulina en Colosenses según la cual en Cristo están todos los tesoros del conocimiento y de la sabiduría $(\mathrm{Col} \mathrm{2,3})^{48}$. Así, Calvino previene la conclusión que de otro modo podría ser sacada, esto es, que Cristo es solo el comienzo -solo una parte- de la construcción, y que el acabamiento de la construcción, su perfección, podría provenir de otro lugar. La vinculación con este pasaje de Colosenses permite, por decirlo así, afirmar que Cristo es el único fundamento, pero bloqueando el minimalismo doctrinal que se podría derivar de ahí. Entonces, afirma Calvino, este pasaje de 1 de Corintios debe ser entendido en el sentido de que el que aprendió a Cristo, "ya se ha graduado en todo el sistema divino de doctrina» $»^{49}$. De este modo, precisamente un pasaje que podría ser usado por los minimalistas, es interpretado por Calvino de un modo que nos hace conscientes de la necesidad de tener tal sistema completo, de buscar todos los tesoros del conocimiento y de la sabiduría. Así, si bien se puede decir que el "fundamento» que hemos recibido es según Calvino doctrinal, se trata no de una doctrina, sino de un sistema doctrinal o, por decirlo de otro modo, de todo un modo de ver el mundo.

Cabe además notar que tal posición no es inusual en su obra. Fuera del contexto exegético que acabamos de comentar, en el contexto polémico del Interim de Ausburgo, Calvino rechaza la idea de que «conservando lo esencial es tolerable perder el resto». Tal posición suena razonable, pero presupone precisamente un tipo de separación entre cosas esenciales y no esenciales que Calvino no hace, una que de hecho ridiculiza «como si Cristo hubiese sido entregado para ser dividido según nuestra

\footnotetext{
$47 \mathrm{CO} 49,357$.

48 CO 49, 354.

49 CO 49, 354.
} 
discreción». Se trata de un texto llamativo por su inequívoca identificación de Cristo con un robusto sistema de práctica y doctrina cristiana. Calvino concede, por supuesto, que la adquisición o recuperación de tal sistema puede tener que ser gradual, pero insiste en que sería un sacrilegio querer partir en dos este "compacto", pues la doctrina cristiana nos ha sido dada para ser gozada de modo completo ${ }^{50}$.

Constituye, por cierto, un significativo punto de continuidad entre Lutero y Calvino, uno que no ha sido notado, que este tipo de defensas de un completo cuerpo doctrinal los lleven con tanta naturalidad a hablar del gozo: «déjanos ser assertores -escribe Lutero a Erasmo- y ser de los que desean las assertiones y se gozan en ellas» ${ }^{51}$.

El balance que se puede sacar de los autores que hemos revisado parece pues inequívoco. Es cierto que si se atiende al número de doctrinas confesadas, la Reforma protestante parece iniciar una tendencia doctrinalmente minimalista. Pero apenas se deja de lado dicha consideración cuantitativa para cambiarla por una cualitativa, puede verse que la forma mentis de los reformadores es opuesta a tal minimalismo, a cualquier búsqueda de una separación radical entre doctrinas fundamentales y otras indiferentes. El gran calvinista holandés Herman Bavinck, en medio de un estudio sobre la búsqueda liberal de una «esencia de la religión», concluye precisamente que «según la Reforma, hay varios puntos en que la Iglesia Católica Romana mezcló el cristianismo original con elementos que le son ajenos; pero la Reforma misma no profesa una abstracta "esencia" del cristianismo, sino que busca restaurar a su debido honor un cristianismo completo, pleno" ${ }^{52}$. Conviene ahora verificar si respecto del campo práctico se puede llegar a conclusiones análogas.

\section{JUSTIFICACIÓN POR LA FE E INDIFERENCIA MORAL}

Los resultados a los que hemos llegado hasta aquí podrían conducir a la conclusión de que si bien se mantiene con la Reforma una concepción relativamente sustantiva del corpus doctrinae, no ocurre lo mismo con la visión de la vida moral. Esta, en efecto, podría verse afectada, de

$50 \mathrm{CO} \mathrm{7,593.}$

51 WA $18,605$.

52 H. Bavinck, "The Essence of Christianity» en Essays on Religions, Science, and Society (Eerdmans, Grand Rapids 2008), 38. 
un modo neutralizante, por al menos dos vías vinculadas a elementos centrales de la Reforma: o bien por la aparente contraposición entre justificación por fe y por obras -que parece volver indiferente el obrar, al menos para la justificación-, o bien por el modo en que se entienda la relación entre la ley y la libertad del cristiano. La literatura secundaria ha abundado aquí en afirmaciones de carácter general que dificultan un juicio sereno. Un estudioso tan destacado como Timothy Wengert, por ejemplo, ha sostenido que la Reforma trataría los adiaphora de un modo radicalmente distinto a la tradición precedente, y para fundamentar tal juicio contrasta el reconocimiento de la existencia de cosas indiferentes entre los reformadores con la negación tomista de que pueda haber actos intencionales individuales de carácter indiferente ${ }^{53}$. Pero comparar lo que los reformadores afirman en general con lo que Tomás de Aquino afirma que vale in individuo difícilmente echa luz sobre la cuestión. Como veremos, una mirada al detalle parece anular dicho contraste. Intentaremos mostrar esto en los dos campos mencionados, atendiendo en primer lugar a la justificación por la fe, y en segundo lugar a la discusión sobre la noción de la «libertad del cristiano».

a) Laxismo moral: una acusación cruzada

Una de las primeras sorpresas que se lleva quien dirige la mirada al problema que aquí tratamos es el hecho de que la acusación en cuestión, que se esté minando el orden moral puesto por Dios en la creación, y mediante expreso recurso a la noción de cosas indiferentes, es en realidad lanzada en el siglo XVI desde los dos lados de la frontera confesional. Esto es, no solo como acusación del catolicismo romano a los reformadores protestantes, sino también en la dirección opuesta. La acusación se encuentra, como veremos, entre las comunes (aunque menos conocidas) objeciones protestantes al pensamiento católico tardomedieval, y se encuentra también en el concilio tridentino, cuya sexta sesión (sobre la justificación) contiene, en el canon XIX, la afirmación de que «si alguien dijere que en el Evangelio no hay ningún precepto más allá de la fe, y que todas las cosas restantes son indiferentes (cetera esse indifferentia) o libres, o que los diez mandamientos no son relevantes (nibil pertinere)

53 T. Wengert, «adiaphora», en H. Hillerbrand (dir.), The Oxford Encyclopedia of the Reformation (Oxford University Press, Oxford 1996) I, 5. 
para los cristianos: sea anatema ${ }^{54}$. Intentaremos aquí aclarar qué motivaba en ambos casos esta acusación. En cualquier caso, el carácter recíproco de la misma ya indica que esta debe ser tomada cum grano salis: piensen lo que piensen los implicados sobre sus respectivos adversarios, unos y otros parecen querer defender algo muy similar.

Cabe, para comenzar, notar que Ireneo había ya rechazado un tipo de comprensión de la centralidad de la fe en tales términos. En el Adversus Haereses se refiere, en efecto, a grupos que sostendrían que como "por la fe y la caridad somos salvos», "todo el resto es indiferente». Significativamente, en el mismo pasaje esta afirmación se mezcla con otra de trasfondo sofista según la cual «es según la opinión de los hombres que unas cosas son llamadas buenas y otras malas, ya que nada es malo por naturaleza ${ }^{55}$. El canon tridentino que hemos citado no llega a atribuir a los reformadores protestantes la posición sofista, pero sí les atribuye el entender la centralidad dada a la fe en términos de una resurrección de la posición gnóstica combatida por Ireneo. Todavía hoy dicho argumento sigue estando vigente entre importantes pensadores católicos, que ponen en duda el levantamiento de este tipo de condenas recíprocas en el contexto del movimiento ecuménico contemporáneo. Al parecer de John Finnis, por ejemplo, se debería privilegiar la perspectiva de los autores del siglo XVI, los cuales comprendían bien lo sostenido por sus adversarios y por lo mismo levantaban tal voz de alerta ${ }^{56}$. Pero en el presente punto el atender a textos de los autores del siglo XVI tal vez puede ser provechoso precisamente por el modo en que parece respaldar el levantamiento de recíprocos anatemas en esta materia. Pues Calvino, quien en su Antídoto a Trento presenta cada artículo que le parece importante acompañándolo de una refutación, cita el canon XIX y su condena de la indiferencia moral, acompañando la cita de un sencillo "amén" ${ }^{57}$. En el campo luterano la situación es idéntica. Martin Chemnitz, el principal de los controversialistas antitridentinos, resuelve la cuestión también de modo sumario, por lo evidente que le parece el hecho de encontrarse

54 Sess. VI, canones de iustificatione, can. 19 (Denzinger 1569).

55 Ireneo, Adversus Haereses. I, 25, 5. Tanto el texto latino como el griego preservan en este pasaje toda la terminología clásica en discusión: indifferentialadiaphora, opinioldoxa, naturalphysei.

56 J. Finnis, Religion and Public Reasons (Oxford University Press, Oxford 2011), 165.

$57 \mathrm{CO} 7,480$. 
ante una caricaturización (calumnia) ${ }^{58}$. Abre su breve comentario simplemente afirmando que «sobre esto no hay entre nosotros controversia alguna ${ }^{59}$. «Esto ha sido refutado ya muchas veces» ${ }^{60} \mathrm{y}$ "tampoco es desconocido por los mismos pontificios» ${ }^{61}$. Lo que sigue a continuación en su texto es una simple repetición del papel desempeñado por la ley moral en la vida del cristiano, repetición que el mismo Chemnitz trata como una tediosa enumeración de puntos obvios. Lo que de estos dos testimonio se puede concluir es sencillo: los textos protestantes dan la razón a la condena católica, pero afirman no encontrarse entre los condenados por ella; y apenas consideran necesario argumentar respecto de dicho último punto, pues no parece haber de la contraparte argumento alguno al cual se pudiera siquiera responder.

Pero como señalamos, la acusación es bidireccional, también dirigida por los reformadores a sus adversarios de los siglos inmediatamente precedentes. La acusación se puede encontrar sumamente temprano en Melanchthon, si bien en un comentario marginal. En efecto, en los loci de 1521 saca esto a colación como ejemplo de la posibilidad de un error conciliar: el Concilio de Constanza habría clasificado las acciones como buenas, malas o indiferentes, siendo que según Mateo 12, 33 solo se dividen en buenas y malas ${ }^{62}$. De un modo más detenido, y en el contexto de una disputación sobre la justificación, Lutero escribe el año 1536 sobre «los escolásticos» que por «ignorancia inventaron que hay ciertas obras ni buenas ni malas, sino intermedias (media) o naturales $»^{63}$. Esta acusación, que pone en el medioevo el origen de una tendencia laxista, y que en concreto la vincula a la discusión sobre lo indiferente, es algo que no solo se encuentra en Lutero, sino que sería repetido todavía un siglo más tarde por escolásticos protestantes como Quenstedt ${ }^{64}$. Al lector de

58 M. Chemnitz, Examen Concilii Tridentini, ed. Preuss (Schlawitz, Berlín 1861), 200. a qué decisión del concilio se refiere Melanchthon; tampoco nuestra propia investigación permite actualmente establecerlo.

63 WA 39/1, 85.

64 Incluso sobrevive hoy, sin recurso expreso a la noción de adiaphora, entre aquellos pensadores protestantes que consideran como lo esencial del escolasticismo el espíritu de síntesis entre una mentalidad bíblica y una extrabíblica con pretensiones 
hoy le puede sorprender tal crítica. No solo parece de facto errónea, sino que parece sorprendente que a los reformadores protestantes les pudiera parecer un punto digno de discusión. En el grado de generalidad con que es formulada la acusación, es por supuesto tanto o más calumniosa que el canon XIX de Trento, pues hace caer un sinnúmero de posiciones medievales bajo un mismo denominador. Pero la preocupación de los reformadores por este punto no debe ser considerada marginal, sino que se vincula a posiciones fundamentales de los mismos: lo que están diciendo es que en la iglesia tardomedieval pudo llegar a haber un desarrollo semipelagiano precisamente porque las exigencias de la ley moral no estaban siendo expuestas de un modo acabado. La posición de los reformadores puede ser fácilmente expuesta en los siguiente términos: si se viera la ley moral en pleno vigor, sin convertir preceptos en meros consejos y sin dar lugar a actos individuales indiferentes, no habría lugar a creer que el hombre es capaz de cumplir con dicha ley por sus propias fuerzas. Es algo más común que los reformadores hagan notar este punto criticando la distinción entre praecepta y consilia evangelica, pero es en ese mismo contexto que debe ser vista la crítica a la noción de actos indiferentes.

Pero esto significa que tiene pleno sentido intentar averiguar si la crítica de facto tiene algún asidero en textos medievales. No esperando en Lutero una discusión sobre la especificación del acto moral, sus editores modernos ofrecen aquí una explicación insuficiente. En una nota al pie del enigmático pasaje que hemos citado, ofrecen un panorama del desarrollo medieval de la cuestión en los siguientes términos: primero habría en san Agustín una transmisión del concepto estoico de adiaphora, en segundo lugar se pasaría a la aceptación escotista de acciones indiferentes in individuo, para finalmente señalar que dicho campo se vio considerablemente ampliado por el ockhamismo, en concreto en Gabriel Biel $^{65}$. Hay algo de verdad en tal descripción de los hechos; pero el texto de Gabriel Biel al que hacen referencia los editores de Lutero no es un texto sobre la especificación del acto moral, que es el lugar en el que se desarrolla usualmente el problema, sino sobre la existencia de obras «naturales» en el sentido de que también sin la gracia puedan ser considera-

de neutralidad. Para un elocuente ejemplo de este tipo de crítica a la "escolástica», véase R. Clouser, The Myth of Religious Neutrality. An Essay on the Hidden Role of Religious Belief in Theories (University of Notre Dame Press, Notre Dame 2005), 98-104.

65 WA 39/1, 85 n. 2. 
das como moralmente buenas ${ }^{66}$. Pero está claro por el pasaje en cuestión que Lutero está en realidad más inserto en una discusión medieval sobre la especificación del acto moral que lo que sería usual pensar: el término media es un frecuente equivalente de indifferentes, establecido ya desde Cicerón como una de las posibles traducciones latinas de adiaphora ${ }^{67}$. Está además claro que dentro de dicha discusión tardomedieval hay un desarrollo por el que crecientemente se afirma la existencia de tales obras indiferentes también en el caso de un acto individual e intencional ${ }^{68}$. Tal desarrollo obedece por lo demás -al menos en parte- a un esfuerzo por mitigar lo que de otro modo parecerían las exigencias excesivas de la ley moral. Es, en efecto, una constante entre los autores que aprueban la idea de acciones individuales intencionales indiferentes el afirmar que la tesis contraria sería "excesivamente dura» (nimis durus), que «estrecha demasiado el camino hacia el reino ${ }^{69}$. El juicio histórico de los reformadores no parece pues errado respecto de tal desarrollo, aunque sí cabe afirmar que es un juicio insuficientemente diferenciado, que omite aquel lado de la tradición medieval con el cual habrían concordado en caso de conocerlo. En Tomás de Aquino, en efecto, se llega a conclusiones plenamente compartibles por los reformadores, de un modo que obliga a cualificar decididamente los juicios de Wengert que hemos citado. Pues tanto la afirmación de la existencia genérica de acciones indiferentes como la negación de su existencia en acciones intencionales interesa a uno y otros, aunque sea por motivos distintos. En ese sentido no es de extrañar que, como ampliamente ha mostrado Reimund Sdzuj, en esta materia la continuidad con el tomismo no constituye una excepción, sino que marcara la norma tanto para los reformadores como para el pensamiento protestante de al menos todo el siglo siguiente ${ }^{70}$.

b) La libertad del cristiano

En un último paso, dirigiremos nuestra atención al problema de la «libertad del cristiano» y el papel de lo indiferente en la discusión sobre

66 Biel, In II Sent., d. 28.

67 Véase De finibus III, 39; III, 53 y III, 58.

68 Véase Duns Escoto, Ordinatio II, d. 41 y Pedro Aureolo II, d. 40.

69 Así G. Biel en II, d. 41, art. 3. Del mismo modo juzga ya Buenaventura, In II Sent, d. 40, q. 3.

70 Véase R. SDzuj, Adiaphorie und Kunst. 91-174. 
la misma. Proviene de Lutero la célebre obra con el título La libertad cristiana, y tras él el tópico se volvió un frecuente componente de las obras dogmáticas del protestantismo. Como parte de dicho desarrollo hay una creciente especificación de los sentidos en que se puede decir que el cristiano, por serlo, es libre: la libertad respecto de la servidumbre de la ley, la libertad para obediencia gozosa y, a continuación, el libre uso de lo indiferente ${ }^{71}$. Lo usual es que dicho último punto, sobre la libertad en torno a lo indiferente, sea el lugar para discutir también aspectos del culto. Así ocurre ya en la mencionada obra de Lutero, que contiene la esperable crítica a los que «descuidan cosas de peso y necesarias para la salvación, generando conflicto sobre liviandades y cosas no necesarias ${ }^{72}$. Si bien los aspectos litúrgicos no son objeto específico del presente artículo, aquí se ofrece una caracterización general de las posiciones de los reformadores, en la medida en que lo que dicen sobre el culto se aplica a las acciones humanas en general y nos permite así en un último paso evaluar la coherencia de su posición.

El mismo Lutero da cuenta de cómo bajo el título de «libertad cristiana» se empiezan tempranamente a cobijar posiciones en realidad rivales de las suyas. En particular los prólogos de sus catecismos son lugar privilegiado para encontrar dichas quejas, las que en parte explican la creciente difusión de literatura catequética en la segunda mitad de la década de 1520. El mismo tipo de preocupación es expresada por Calvino $^{73}$, lo cual debiera ser motivo suficiente para no vincularla exclusivamente a los abusos de grupos radicales en Wittenberg, sino también a problemas que los reformadores diagnostican entre sus propios fieles. Las impresiones recogidas en una visita pastoral de los teólogos de Wittenberg a los territorios sajones entre 1526 y 1527 son iluminadoras al respecto. El crítico análisis de la formación recibida por el pueblo se encuentra en un texto redactado por Melanchthon, un conjunto de artículos publicados en 1527 sin su consentimiento. La versión alemana del año siguiente, Unterricht der Visitatoren, es más extensa y contiene además modificaciones hechas por Lutero, junto a un prefacio del mis-

71 En ese orden lo tratan, por ejemplo, Calvino en Inst. III, 19, 1-8 y Melanchthon, CR 26, 25.

72 WA 7, 69-70. La afirmación solo se encuentra en la versión latina del tratado, que sobre todo en las últimas páginas difiere significativamente de la alemana.

73 Inst. III, 19, 1. 
$\mathrm{mo}^{74}$. Se trata pues de un texto representativo no solo de Melanchthon, sino de la teología wittenbergense en general. Y ahí, si bien hay un reconocimiento tácito de la existencia de acciones genéricamente indiferentes, el acento cae de modo inequívoco en la crítica a quienes, acentuando dicha indiferencia, generan controversia en torno a las mismas. La crítica a tradiciones humanas que no son nocivas, escribe Melanchthon en la versión latina, «solo tiene por resultado que el vulgo aprenda a despreciar tanto las leyes humanas como las divinas» ${ }^{75}$. Quienes solo hablan de la libertad cristiana en relación a las cosas indiferentes, dejando de lado el necesario énfasis en los otros grados de tal libertad «tragan el camello y cuelan el mosquito» ${ }^{76}$. Parece así claro que lo que tenemos por delante es la idea de que existe un amplio campo de tipos de acciones genéricamente indiferentes, pero que en concreto ninguna lo es, pues el acto concreto siempre estará especificado por factores como el amor o el desdén: el carácter indiferente de una tradición humana no permite que la rechacemos simpliciter. Ese tipo de lógica respecto del actuar humano en general es la que está siendo aplicada aquí al culto, llevando a que la afirmación general de que muchos elemenos del mismo sean indiferentes, luego esté fuertemente cualificada cuando se trata de introducir cambios concretos.

Si dirigimos la mirada a Calvino, este habla sobre el conocimiento de esta "libertad del cristiano» como una cuestión "sumamente necesaria», como un "apéndice de la justificación» (appendix est iustificationis) ${ }^{77}$. Tal como Melanchthon, explica que se puede hablar de tal libertad en distintos niveles, varios de los cuales no se vinculan con lo indiferente. Pero cuando llega al punto en que sí toca tal conexión, el interés de Calvino por la noción se hace notar desde la misma elección de los términos a utilizar. No solo en la referencia al griego adiaphoroi ${ }^{8}$, sino en el hecho de que donde habla de cosas indifferentes en la edición latina, la edición

\footnotetext{
74 Se trata de un texto significativo además por la controversia que genera respecto de la doctrina de la penitencia, y por su papel en el surgimiento de la primera controversia antinomista dentro del protestantismo. Al respecto véase T. WENGERT, Law and Gospel. Phipil Melanchthon's Debate with John Agricola of Eisleben over Poenitentia (Baker, Grand Rapids 1997).

75 CR 26, 23.

76 Esta alusión a Mateo 23,24 se encuentra en CR 26:23 y 26.

77 Inst. III, 19, 1.

78 Inst. III, 19, 7.
} 
francesa (de la pluma del mismo Calvino) completa diciendo «las cosas que no son en sí buenas ni malas» ${ }^{79}$. Por si alguna duda cabe al respecto, tal lenguaje nos debiera recordar cuán claramente insertos están los reformadores en discusiones clásicas. En un punto de la discusión, Calvino concede que existen cosas indiferentes, "pero siempre que se las use de modo indiferente» ${ }^{80}$-un claro eco estoico ${ }^{81}$. La discusión de Calvino se inicia con la preocupación respecto del culto, pero se expande luego a un rango mayor de posibles acciones indiferentes, como la acumulación de riquezas. Saber lo que es la libertad en este campo, Calvino lo tiene por algo necesario para la tranquilidad de las conciencias y para erradicar la superstición ${ }^{82}$. En efecto, parece haber un énfasis mayor que en Melanchthon respecto de la importancia de reconocer la incapacidad de las ceremonias para aplacar a Dios: «a muchos ineptos les extraña», escribe, su preocupación por la libertad respecto de cosas menores como el uso de comidas o vestimentas, "pero hay en esto algo de importancia mayor que lo que generalmente se cree» ${ }^{83}$. Tras estas observaciones ofrece un detenido análisis del tipo de tormentos de conciencia sufridos por quien tiene por impuro algo en realidad indiferente ${ }^{84}$. Pero a esta consideración por la libertad respecto de lo indiferente sigue de inmediato un énfasis que lo acerca a las preocupaciones de Melanchthon que ya hemos visto: una preocupación por la ostentación respecto de lo indiferente, por la acumulación de bienes indiferentes, por el trato con lo indiferente de un modo que no lleva a edificación. «Los pecados del presente siglo», juzga Calvino, "se encuentran más presentes en este punto» que en la ignorancia respecto de la naturaleza de lo indiferente ${ }^{85}$.

Aunque el Interim de Ausburgo traería pronto diferencias entre ellos, podemos aquí ver que hay una forma mentis común a los reformadores también respecto del papel de lo indiferente en el culto. Esto es, junto a un énfasis en la inutilidad de ceremonias para ganar el favor de Dios y junto, en ese sentido, con la reducción de muchas de ellas a una

\footnotetext{
79 Inst. III, 19, 13.

80 Inst. III, 9, 9.

81 Véase Epícteto, Diss. 2, 5, 7.

82 Inst. III, 19, 7.

83 Inst. III, 19, 7.

84 Inst. III, 19, 8.

85 Inst. III, 19, 9.
} 
condición indiferente, hay simultáneamente un llamado predominante a no romper con ellas, en calidad de indiferentes seguir cultivándolas. Esto implica que la libertad respecto de lo indiferente no solo está externamente delimitada, en el sentido de que algo no esté ni ordenado ni prohibido, sino solo permitido en las Escrituras. A tal delimitación externa se suma una interna, al considerarse si el acto concreto que es indiferentemente permitido contribuirá a conservar en amor el vínculo de la paz ${ }^{86}$. Pero el que cada acto concreto tenga que ser sometido a tal evaluación implica que in concreto nada es indiferente. En la polémica generada por el Interim, Flacius sostendría que tampoco respecto de las cosas indiferentes es posible ceder en un armisticio como el entonces experimentado. Según su célebre afirmación, que adquiere rango confesional en el posterior Libro de Concordia, «en una situación de confesión o escándalo, nada es indiferente» ${ }^{87}$. A la luz de lo visto, tal conclusión no convierte a Flacius en un aislado representante de una posición radical, sino en un confesor más de una tradicional posición compartida también por otros reformadores.

\section{Conclusiones}

Una superficial mirada lexicográfica podría permitir establecer un significativo aumento del término adiaphora en el siglo XVI. Pero el masivo recurso a este término griego no debe engañarnos respecto de la continuidad de la discusión: por siglos había habido abundante discusión sobre los facta media o indifferentes, y las posiciones defendidas por los reformadores protestantes deben ser entendidas como capítulos dentro de dicha larga tradición. Pero acentuar tal continuidad solo podría tener sentido en caso de que hubiera antes de la Reforma una única posición relevante. En el caso de las cosas indiferentes ciertamente no es así, sino que hay en el trasfondo de la Reforma una variada gama de corrientes, entre las cuales se encuentra una creciente afirmación tardomedieval de la existencia de acciones intencionales indiferentes. En tal contexto, lejos de poder leerse la Reforma como una liberación en medio de un predominante ambiente tomista, en este punto es una vuelta a una posición

86 Tomo esta distinción entre especificación externa e interna de B. Verkamp, «The Limits upon Adiaphoristic Freedom: Luther and Melanchthon", en Theological Studies 36, 1 (1975), 52-76.

87 Libro de Concordia, solida declaratio, $\mathrm{x}$. 
grosso modo similar a la de Tomás de Aquino, en medio de un ambiente predominantemente abierto a la idea de acciones moralmente neutras.

Puede también ser útil recordar que, junto a su temprano uso estoico, la noción de adiaphora desempeña también un temprano papel entre los cínicos, para designar aquellas convenciones sociales por romper con las cuales el cinismo es célebre. En su polémica con Lutero, Tomás Moro lo describía precisamente como un cínico, como alguien para el que «todo es libre, alguien al que no le importa dónde, cuándo ni cómo sacrifica, de día o de noche, en luz o tinieblas, ebrio o sobrio» ${ }^{88}$. No es extraño que surgiera tal impresión al ver el grado de decisión con que se llevaba adelante la Reforma. Pero parece claro que la autocomprensión de los reformadores se dirige en una dirección opuesta, que también condena el rupturismo cínico. Según hemos visto, Calvino incluso llega a considerar que ese es el punto en que más se concentraría el pecado de su siglo respecto de las cosas indiferentes, incluso más que en las prácticas supersticiosas respecto de cosas indiferentes.

Estas son las conclusiones que cabe sacar respecto de la discusión general sobre las acciones. Pero así como en la escolástica tardía había un ambiente de creciente apertura a la idea de neutralidad moral, en el contexto humanista circulaban análogas ideas respecto del campo doctrinal. También ese es un desarrollo al que la Reforma, por lo que hemos podido ver, se opone, a pesar de los muchos puntos específicos en que pueda constatarse influencia del humanismo renacentista sobre la metodología teológica de algunos de los reformadores ${ }^{89}$. Con todo, que se haya gestado la impresión de que los reformadores representen en el campo moral o doctrinal una versión reducida del cristianismo, que vuelva indiferente una parte significativa del cuerpo doctrinal o de la enseñanza moral, es un fenómeno explicable no solo por los malentendidos que hemos podido constatar en algunas de las acusaciones recíprocas. Después de todo, no solo parece haber en ellos un enfático intento por mostrar la fe cristiana como poseída de un centro muy claramente delimitado, sino que tal mensaje es muchas veces presentado mediante el recurso a partículas excluyentes (los solas de la Reforma), que parecen

88 Tomás Moro, Responsio ad Lutherum en The Complete Works of St. Thomas More 5/1 (Yale University Press, New Haven y Londres, 1969), 418.

89 H. Reventlow, The Authority of the Bible, 74-90, ha mostrado la influencia erasmista sobre una figura tan central para la Reforma, como Martín Bucero. 
volver indiferente todo lo que no está incluido dentro de ese aparentemente reducido conjunto. Pero aquí puede venir al caso recordar las sabias palabras de Kierkegaard sobre la Reforma no como una norma, sino como un correctivo: en tanto se entienda la Reforma como un golpe de timón dentro de la gran tradición precedente, pero continuando dicha tradición en el intento por pensar no solo aspectos específicos, sino la totalidad de la realidad, no habrá confusión, se reconocerá que la afirmación solus Christus no es una apuesta por una parte de la realidad, sino una confesión concentrada de todo lo que debe ser creído. En caso contrario, si el correctivo se toma por norma, no es extraño que la Reforma acabe siendo vista como inauguración, en el campo moral o doctrinal, de diversas posiciones «indiferentistas». Pero, por lo que hemos visto en torno a la noción de adiaphora, tal conclusión sería deficiente no solo por un dudoso marco general de interpretación, sino por chocar con virtualmente toda la evidencia textual específica. 
574 | Manfred Svensson

Resumen: el artículo presenta un análisis de la noción de cosas indiferentes (adiaphora) en el contexto de la Reforma protestante. La literatura precedente sobre dicha noción en el contexto de la Reforma ha estado concentrada en el papel desempeñado por la misma en relación a la diversidad litúrgica. Aquí se aborda, en cambio, de modo primordial la cuestión de si acaso entre los reformadores hay una extensión de la noción de adiaphora al campo doctrinal y al moral.

Palabras clave: Adiaphora, indiferencia, minimalismo doctrinal, neutralidad moral, Lutero, Calvino, Melanchthon.

Abstract: This article presents an analysis of the notion of indifferent things (adiaphora) in the context of the Protestant Reformation. The previous literature on this notion in the context of the Reformation has focused on the role the Reformation has played with regard to liturgical diversity. Here, however, the focus is primarily on the question of whether among reformers there is an extension of the notion of adiaphora to the doctrinal and moral field.

Keywords: Adiaphora, indifference, doctrinal minimalism, moral neutrality, Luther, Calvin, Melanchthon. 